\title{
Antiferromagnetic vs ferromagnetic interactions and spin-glass-like behavior in ruthenates
}

\author{
P.Ravindran,f R.Vidya, P.Vajeeston, A.Kjekshus, and H.Fjellvåg \\ Department of Chemistry, University of Oslo, Box 1033, Blindern, N-0315, Oslo, Norway \\ B.C. Hauback \\ Institute of Energy Technology, P.O.Box 40, Kjeller, N-2007, Norway
}

(Dated: November 1, 2018)

\begin{abstract}
We have made a series of gradient-corrected relativistic full-potential density-functional calculation for Ca-substituted and hole-doped $\mathrm{SrRuO}_{3}$ in para, ferro, and $A$-, $C$-, and $G$-type antiferromagnetic states. Magnetic phase-diagram data for $\mathrm{Sr}_{1-x} \mathrm{Ca}_{x} \mathrm{RuO}_{3}$ at $0 \mathrm{~K}$ are presented. Neutron diffraction measurement combined with total energy calculations show that spin-glass behavior with short-range antiferromagnetic interactions rules in $\mathrm{CaRuO}_{3}$. The substitution of Sr by $\mathrm{Ca}$ in $\mathrm{SrRuO}_{3}$ decreases the ferromagnetic interaction and enhances the $G$-type antiferromagnetic interaction; the $G$-AF state is found to stabilize around $x=0.75$ consistent with experimental observations. Inclusion of spin-orbit coupling is found to be important in order to arrive at the correct magnetic ground state in ruthenates.
\end{abstract}

PACS numbers: 75., 81.05.Je, 75.10.Nr, 71.20.-b

Ever since unconventional superconductivity was observed in $\mathrm{Sr}_{2} \mathrm{RuO}_{4}$ [1], ruthenates have attracted much interest. Substitution [2] of the smaller $\mathrm{Ca}$ for $\mathrm{Sr}$ in $\mathrm{Sr}_{2} \mathrm{RuO}_{4}$ leads to an antiferromagnetic (AF) Mott insulator with a staggered moment of $S=1$. Coexistence of ferro- and antiferromagnetic fluctuations in $\mathrm{Ca}_{2} \mathrm{RuO}_{4}$ and competition between $p$ - and $d$-wave superconductivity in $\mathrm{Sr}_{2} \mathrm{RuO}_{4}$ have also been reported [3]. Recently coexistence of magnetism and superconductivity is discovered [4] in ruthenium-based layered cuprates such as $R \mathrm{RuSr}_{2} \mathrm{Cu}_{2} \mathrm{O}_{8}(R=\mathrm{Eu}, \mathrm{Gd})$. Lee et al. [5] reported the existence of a pseudogap $(\mathrm{PG})$ in $\mathrm{BaRuO}_{3}$ reminiscent of the $\mathrm{PG}$ in high- $T_{c}$ superconductors. As in $f$-electron-based intermetallics, non-Fermi-liquid (NFL) behavior has recently been observed in $\mathrm{La}_{4} \mathrm{Ru}_{6} \mathrm{O}_{19}[6]$. Critical magnetic fluctuations associated with metamagnetism is reported for $\mathrm{Sr}_{3} \mathrm{Ru}_{2} \mathrm{O}_{7}$ [7] and a metallic $\mathrm{AF}$ phase with temperature induced insulator-to-metal transition is found for $\mathrm{Ca}_{3} \mathrm{Ru}_{2} \mathrm{O}_{7}$ [8].

Poorly metallic NFL-behaving $\mathrm{SrRuO}_{3}$ is the only known ferromagnetic $\left(\mathrm{F} ; T_{C}=160 \mathrm{~K}\right) 4 d$ transition-metal oxide [9, 10]. $\mathrm{CaRuO}_{3}$ is also metallic, but experimental and theoretical studies conclude contradictory regarding the nature of the magnetic ground state (e.g., AF [9, 11, 12], nearly $\mathrm{F}$ [13], exchange enhanced paramagnetic $(\mathrm{P})$ 14], Curie-Weiss $\mathrm{P}$ [15], verge of $\mathrm{F}$ instability [16], spin glass (SG) [17] etc.), and this controversy is not settled yet. Some of these reports indicate a lack of long-range magnetic order whereas others suggest evidence for an AF ground state with a Néel temperature $\left(T_{N}\right)$ of $\sim 110 \mathrm{~K}[11]$. The striking difference in the magnetic properties of $\mathrm{SrRuO}_{3}$ and $\mathrm{CaRuO}_{3}$ makes magnetic

*Electronic address: ponniah.ravindran@kjemi.uio.no; URL: http: //folk.uio.no/ravi phase diagram studies on $\mathrm{Sr}_{1-x} \mathrm{Ca}_{x} \mathrm{RuO}_{3}$ quite interesting.

$\mathrm{CaRuO}_{3}$ and $\mathrm{SrRuO}_{3}$ are isostructural and isoelectronic, differing structurally only in the degree of the small orthorhombic distortion (lattice constants within $2 \%$ ). Hence, insight into the magnetic properties of these compounds is expected to increase the general knowledge on magnetic phenomena in perovskite oxides, and ruthenates in particular. The density-functional calculations 16, 18 for $\mathrm{CaRuO}_{3}$ which conclude with "verge of $\mathrm{F}$ instability" have the weakness that AF interactions were not considered. In this letter, we present the intriguing result that $S G$ behavior with short-range AF interaction occurs in metallic $\mathrm{CaRuO}_{3}$.

The present full-potential linear-muffin-tin orbital 19] (FPLMTO) calculations are all-electron, and no shape approximation to the charge density or potential has been used. The basis functions, charge density and potential were expanded in spherical harmonic series inside the muffin-tin spheres and in Fourier series in the interstitial regions. The calculations are based on the generalizedgradient-corrected (GGA) density-functional theory as proposed by Perdew et al. [20]. Spin-orbit (SO) terms are included directly in the Hamiltonian matrix elements for the part inside the muffin-tin spheres. The basis set contained semi-core $4 p$ and valence $5 s, 5 p$, and $4 d$ states for $\mathrm{Sr}, 4 s, 4 p$, and $3 d$ states for Ca, $5 s, 5 p$, and $4 d$ for Ru, $2 s, 2 p$, and $3 d$ states for $\mathrm{O}$, and $4 s, 4 p$, and $3 d$ states for $\mathrm{K}$. All orbitals were contained in the same energy panel. The self consistency was obtained with $284 \mathbf{k}$ points in the irreducible part of the first Brillouin zone for the orthorhombic structure and the same density of $\mathbf{k}$ points were used for the cubic structure as well as for the supercells. Structural parameters for $\mathrm{Sr}_{1-x} \mathrm{Ca}_{x} \mathrm{RuO}_{3}$ are taken from the X-ray diffraction data reported by Kobayashi et al. 21]. Details about the experimental reexamination of $\mathrm{CaRuO}_{3}$ will be presented in the full account of our 
TABLE I: Total energy (relative to the lowest energy state; in meV/f.u.) for $\mathrm{CaRuO}_{3}$ in $\mathrm{P}, \mathrm{F}$, and $A-, C$-, and $G$-AF states with orthorhombic and undistorted cubic (designated cubic) structures.

\begin{tabular}{lccccl}
\hline \hline Method & $\mathrm{P}$ & $\mathrm{F}$ & $A-\mathrm{AF}$ & $C-\mathrm{AF}$ & $G-\mathrm{AF}$ \\
\hline GGA+SO & 26 & 19.8 & 6 & 4 & 0 \\
GGA+SO (cubic) & 1307 & 1249 & 1292 & 1299 & 1303 \\
GGA & 38 & 0 & 9 & 10 & 7 \\
\hline \hline
\end{tabular}

findings [22].

There are three possible magnetic arrangements according to the interplane and intraplane couplings in perovskite oxides. (i) With interplane AF coupling and intraplane $\mathrm{F}$ coupling the $A$-AF structure arises. (ii) The opposite arrangement with interplane $\mathrm{F}$ coupling and intraplane AF coupling is called $C$-AF structure. (iii) If both the inter- and intraplane couplings are AF the $G$ $\mathrm{AF}$ structure arises. In the $G$-type $\mathrm{AF}$ lattice, each $\mathrm{Ru}$ atom will be surrounded by six Ru neighbors whose moments are antiparallel to that of the chosen reference atom. The details about the calculations on magnetic and excited state properties can be found elsewhere [23]. We have placed the magnetic moment direction along [001] in all calculations to comply with the out-of-plane easy axis observed for $\mathrm{Sr}_{1-x} \mathrm{Ca}_{x} \mathrm{RuO}_{3}$ [24]. The energy differences between the various magnetic configurations are very small and extreme computational accuracy is therefore needed. The theoretical method used in the present study is capable of reproducing energy differences of the order of $\mu \mathrm{eV}$ [25].

The calculated total energy for $\mathrm{CaRuO}_{3}$ from the GGA calculation with $(\mathrm{GGA}+\mathrm{SO})$ and without $\mathrm{SO}$ coupling are given in Table 1 relative to the corresponding ground state. From this table it is clear that the $G-\mathrm{AF}$ phase is the ground state for $\mathrm{CaRuO}_{3}$. The total energy differences between the different AF phases are as already mentioned very small. If we disregard the orthorhombic distortion, the $F$ phase is seen to have the lowest energy indicating that strong magneto-elastic couplings are present in $\mathrm{CaRuO}_{3}$. We also see large energy gains when we include the orthorhombic distortion in the calculation. The $\mathrm{F}$ phase is found to be the ground state with $7 \mathrm{meV} /$ f.u. lower energy than the $G$-AF phase when $\mathrm{SO}$ interactions are neglected. Hence, SO coupling appears to play an important role in deciding the magnetic properties of ruthenates.

The calculated magnetic moment at the $\mathrm{Ru}$ site in $\mathrm{SrRuO}_{3}$ is $0.92 \mu_{B}$ /atom is in good agreement with $0.85 \mu_{B}$ /atom obtained from magnetization measurements [9]. Goodenough [26] has argued that, in general, a spontaneous change between $\mathrm{F}$ and $\mathrm{AF}$ states are expected when $\mu_{F} \approx \mu_{A F}$, the stable magnetic phase being the one with the highest atomic moment. Our findings are consistent with this viewpoint in that the larger magnetic moments are found for the stable magnetic configuration of $\mathrm{Sr}_{1-x} \mathrm{Ca}_{x} \mathrm{RuO}_{3}$ (viz., for $x<0.724$ the $\mathrm{F}$ phase

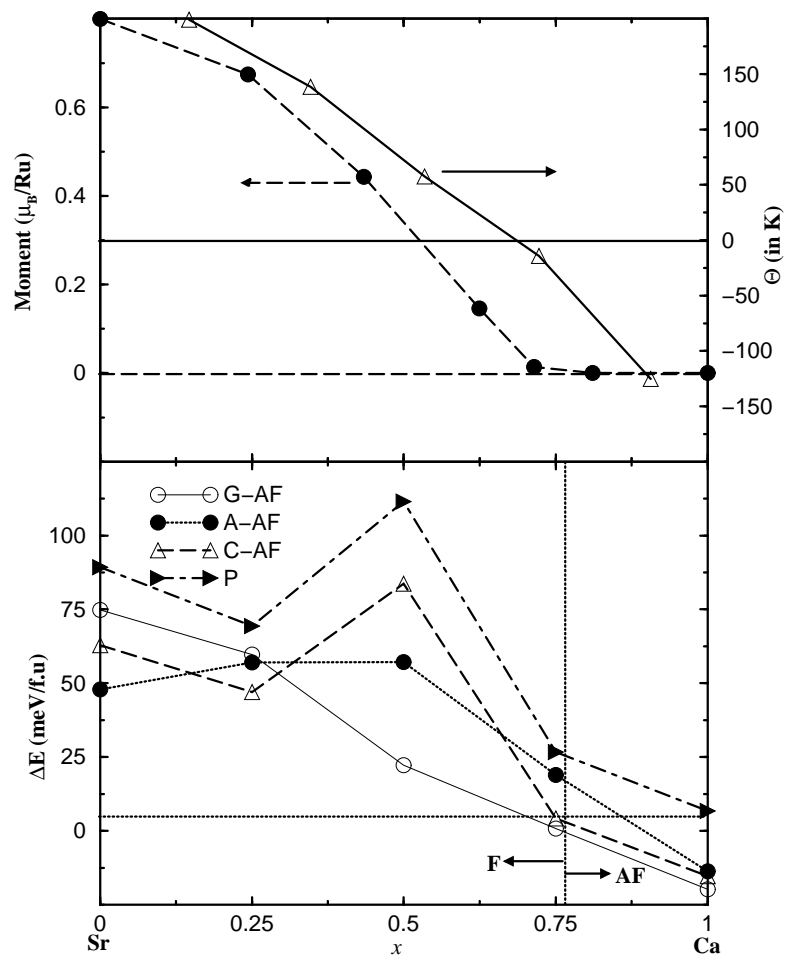

FIG. 1: Magnetic phase diagram data for $\mathrm{Sr}_{1-x} \mathrm{Ca}_{x} \mathrm{RuO}_{3}$. Energies are given with respect to the total energy for the $\mathrm{F}$ phase.

has highest magnetic moment and for $x>0.724 G$-AF has the highest moment). The hyperfine field at the $\mathrm{Ru}$ site in $G$-AF state $\mathrm{CaRuO}_{3}$ is $263 \mathrm{kOe} / \mu_{B}$ in agreement with the experimental value of $222 \pm 50 \mathrm{kOe} / \mu_{B}$ obtained from Knight shift measurements 27.

The experimentally found spontaneous magnetic moment 13] and Weiss temperature 28] for $\mathrm{Sr}_{1-x} \mathrm{Ca}_{x} \mathrm{RuO}_{3}$ are given in Fig. 1 along with the calculated total energy difference relative to that of the $\mathrm{F}$ phase for the various magnetic configurations.

An increase in $x$ decreases the $\mathrm{F}$ interaction and increases the G-AF interaction. Because of the smaller ionic radius of $\mathrm{Ca}$ compared with $\mathrm{Sr}(0.99$ vs $1.18 \AA$ ), tilting of the octahedra occur when one substitute $\mathrm{Ca}$ for $\mathrm{Sr}$ in $\mathrm{SrRuO}_{3}$ which in-turn affect the magnetic properties. Fig. 1 1 shows that the $\mathrm{F}$ to $G$-AF transition takes place around $x=0.75$ both experimentally and theoretically. This confirms the presence of AF interactions in $\mathrm{CaRuO}_{3}$. One of the interesting aspects of Fig. 1 is that at $x=0.5$, there is large gain in the total energy of $\mathrm{F}$ phase compared with $\mathrm{P}, A$ - and $C$-AF phases. DOS analyses show that the $\mathrm{F}$ phase of $\mathrm{Sr}_{0.5} \mathrm{Ca}_{0.5} \mathrm{RuO}_{3}$ is nearly half-metallic with a gap of $0.73 \mathrm{eV}$ in the majority spin channel which gives extra contribution to the stability. Moreover, $E_{F}$ is at peaks in the DOS curves of the P, $A$ - and $C$-AF phases (which further contribute to the enhanced energy difference relative to the $\mathrm{F}$ state).

The lower total energy for the $G$-AF state for $x>$ 0.724 compared with the other magnetic configurations 


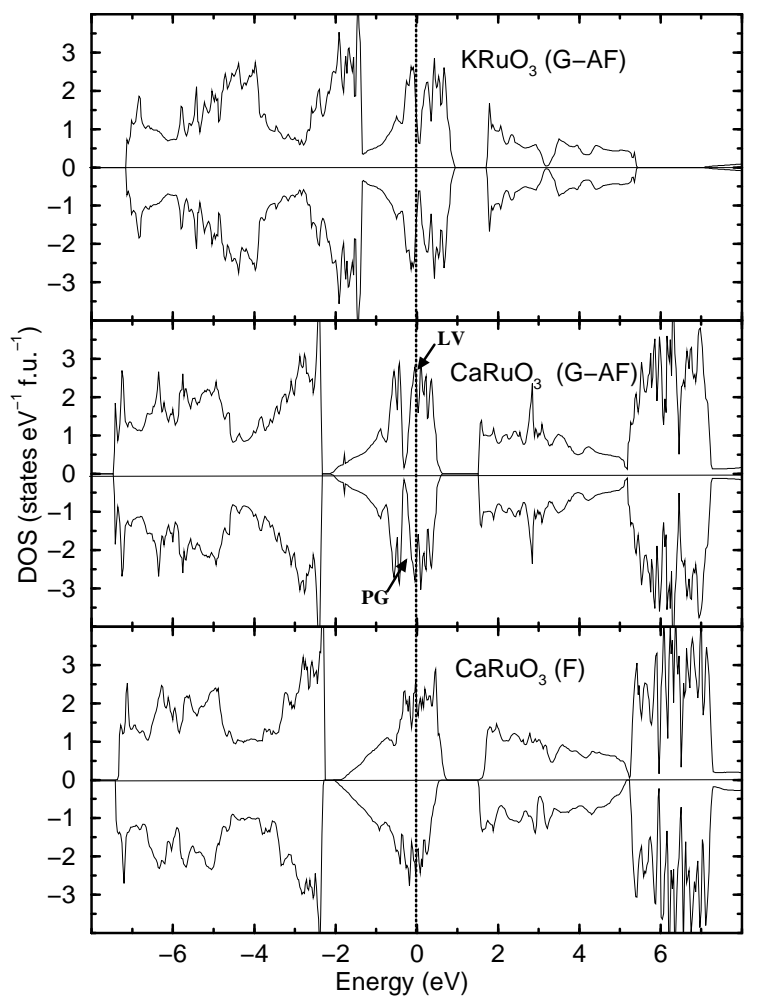

FIG. 2: Total DOS for $\mathrm{CaRuO}_{3}$ in $\mathrm{F}$ and $G$-AF states and hypothetical $\mathrm{KRuO}_{3}$ in $G$-AF state. Fermi level is set to zero.

can be understood as follows. The spin-projected total DOS curve for $\mathrm{CaRuO}_{3}$ in the $\mathrm{F}$ and $G$-AF phases are shown in the lower and middle panel of Fig. 2. In the $\mathrm{F}$ case $E_{F}$ is at a peak-like feature in the DOS curve. This infers an instability to the lattice. However, for the $G$-AF phase a deep valley-like (PG-like) feature appears in the DOS curve. This will give an extra contribution to lattice stability [29] and hence $\mathrm{CaRuO}_{3}$ is expected to stabilize in the $G$-AF phase. The calculated excited-state properties such as reflectivity, O $K$-edge spectra and XPS spectra for $\mathrm{CaRuO}_{3}$ in the $G$-AF phase are found to be in good agreement with experimental spectra (see Fig. 3), indicating that the ground state is correctly assigned. The creation of a $P G$-like feature on introduction of $G-A F$ ordering gives an extra contribution to the band energy term of the total energy ( $66 \mathrm{meV} / f . u$.) which stabilizes the $G$-AF phase over the $F$ phase. However, our neutron powder diffraction diagrams at 298 and $8 \mathrm{~K}$ show no sign of extra Bragg reflections at $8 \mathrm{~K}$, which should have been the proof of long-range AF ordering. (A full account of the neutron diffraction findings will be given in the forthcoming article 222].) Hence, the magnetic exchange interactions must have only short-range influence. Closer examination of the DOS curve for the $G$-AF phase shows that $E_{F}$ is located on a shoulder of a peak-like feature (an unfavorable condition for stability) with a local valley (LV, see Fig. 2) situated just $40 \mathrm{meV}$ above $E_{F}$. This may explain why there is no established long-range magnetic ordering in this material. Recent magnetiza-
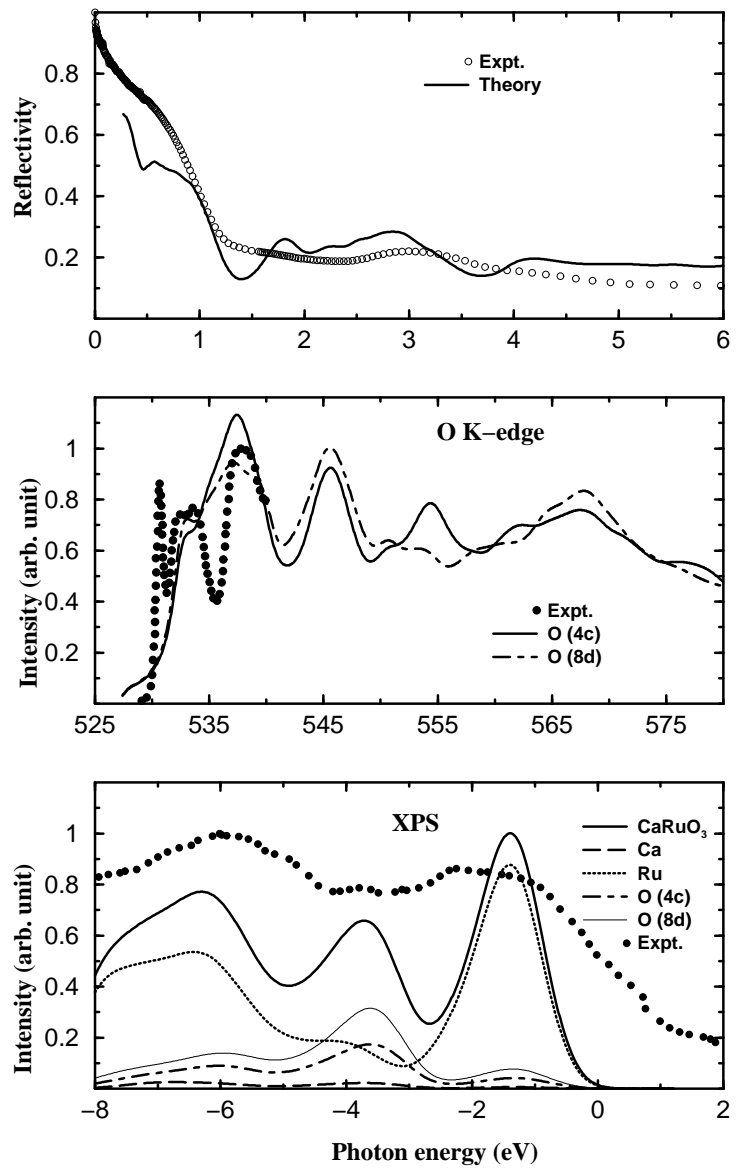

FIG. 3: Calculated valence band XPS, O $K$-edge XANES, and optical reflectivity spectrum for $G$-AF state $\mathrm{CaRuO}_{3}$. Experimental XPS, XANES and reflectivity spectra are taken from Refs. 30, 31.

tion measurements [17] show distinctions between in the zero-field-cooled and field-cooled characteristics that may indicate SG behavior. So, we conclude that $\mathrm{CaRuO}_{3} \mathrm{ex}$ hibits short-range AF ordering which manifests itself in $S G$ behavior at low temperature.

Rigid-band-filling analysis show that an addition of 0.136 electrons/f.u. will bring $E_{F}$ to $\mathrm{LV}$ with the expected effect of long-range $G$-AF ordering. In fact, long-range magnetic ordering has been observed for $\mathrm{CaRu}_{1-x} \mathrm{Rh}_{x} \mathrm{O}_{3}$ with $x>0.015$ where Rh acts as an electron donor for $\mathrm{CaRuO}_{3}$ [32]. Rigid-band-filling analysis also show that removal of exactly one electron from $\mathrm{CaRuO}_{3}$ will bring $E_{F}$ to a PG. In order to test this possibility we have made additional calculations for hypothetical $\mathrm{KRuO}_{3}$ with the structural parameters for $\mathrm{CaRuO}_{3}$. Consistent with the rigid-band-filling findings $E_{F}$ is found at PG for the $G$-AF phase of hypothetical $\mathrm{KRuO}_{3}$ (see upper panel of Fig. 2). When $E_{F}$ is at PG, all bonding states would be filled and all antibonding states empty, implying extra contributions to stability. Total energy calculations show that the $G$-AF phase is $21.7 \mathrm{meV} /$ f.u. lower in energy than the $\mathrm{F}$ phase for hypothetical $\mathrm{KRuO}_{3}$, viz. a larger difference than that 
for $\mathrm{CaRuO}_{3}$ indicating that one really should expect $G$ AF long-range ordering in $\mathrm{KRuO}_{3}$ at low temperature. Further the $G$-AF phase is $38.6 \mathrm{meV} /$ f.u. lower in energy than the $\mathrm{P}$ phase for hypothetical $\mathrm{KRuO}_{3}$ indicating that $T_{N}$ should be reasonably high. It is worth to recall that according to Goodenough 33 the sign of the transfer integral for $180^{\circ}$ cation-anion-cation superexchange interactions between octahedral-site cations are predicted to give $\mathrm{AF}$ for $\mathrm{Ru}^{5+} \mathrm{O}_{-} \mathrm{Ru}^{5+} \cdot \mathrm{Ru}^{5+}$ ions with enhanced AF interaction has recently been established for $\mathrm{CaRu}_{0.95} \mathrm{Cu}_{0.05} \mathrm{O}_{3}$ [34]. Moreover, the change in the oxidation state of $\mathrm{Sr}_{3} \mathrm{Ru}_{2} \mathrm{O}_{7}$ from $\mathrm{Ru}^{4+}$ to $\mathrm{Ru}^{5+}$ by fluorine addition stabilizes $G-\mathrm{AF}$ ordering 35 . Oxygen nonstochiometry and hole doping can accordingly bring $E_{F}$ toward PG and stabilize the $G$-AF ordering. This may explain why long-range AF ordering has been reported for some experimentally studied $\mathrm{CaRuO}_{3}$ samples 11 and $\mathrm{Na}$ doped $\mathrm{CaRuO}_{3}$ 36.

In the SG state the moments are arranged in a certain equilibrium oriented pattern, but without long-range order. Some characteristics of SG materials are: (i) Existence of local moment as we have found with spinpolarized calculations. (ii) No magnetic Bragg scattering at low temperature as our neutron diffraction study show. (iii) A history-dependent magnetic response as the recent magnetization measurements [17 have shown. (iv) Both long-range and short-range terms in the Hamiltonian [37. In accordance with our calculations we believe that short-range nearest-neighbor AF interactions are dominant in $\mathrm{CaRuO}_{3}$.

Two important ingredients necessary to produce SG behavior are frustration and partial randomness of the interaction between the magnetic moments 38. Owing to the small total energy differences between the AF and $\mathrm{F}$ phases of $\mathrm{CaRuO}_{3}$ (see Table and Fig. 1), there will be a competition among the different $\mathrm{AF}$ and $\mathrm{F}$ interactions, in the sense that no single configuration of the the moments is uniquely favored by all interactions (viz. frustration). In other words, the AF moments can arrange themselves randomly in small domain-like regions with minimal loss in energy. The $\mathrm{Ru}$ ions which are responsible for the magnetic properties of $\mathrm{CaRuO}_{3}$ are in a strictly periodic order. Hence, structural disorder can not be responsible for the SG behavior. The SG state of $\mathrm{CaRuO}_{3}$ is characterized by a predominant AF situation. AF systems with SG-like transition have been observed in ruthenates with pyrochlore-like structures such as $R_{2} \mathrm{Ru}_{2} \mathrm{O}_{7}$ for which recent experimental results indicate that the atomic arrangement does not participate in the P-to-SG transition which is solely associated with the $\mathrm{Ru}$ moments 39]. The small energy differences between the different $A F$ states bring disordering of the moments and hence $S G$ behavior to $\mathrm{CaRuO}_{3}$ at low temperature. In fact, $\mathrm{SG}$ behavior has been found for $\mathrm{CaRuO}_{3}$ doped with small amounts of Sn 40] or Rh [32]. The calculated excited state properties for $\mathrm{CaRuO}_{3}$ in the $G$-AF state are in good agreement with the experimental measurements indicating that the electronic structure does not differ significantly between the $\mathrm{SG}$ and $G$-AF phases.

We conclude that $\mathrm{CaRuO}_{3}$ exhibits short-range AF interaction with SG behavior. The hitherto hypothetical $\mathrm{KRuO}_{3}$ is expected to exhibit long-range $G$-AF ordering. We have demonstrated that the relative strengths of the $\mathrm{F}$ and $\mathrm{AF}$ exchange interactions can be varied by varying the Ca content of $\mathrm{Sr}_{1-x} \mathrm{Ca}_{x} \mathrm{RuO}_{3}$.

PR is grateful to the Research Council of Norway for financial support. Part of these calculations were carried out on the Norwegian supercomputer facilities. 
[1] Y. Maeno, H. Hashimoto, K. Yoshida, S. Nishizaki, T. Fujita, J.G. Bednorz, F. Lichtenber, Nature 372 (1995) 532 .

[2] S. Nakatsuji, Y. Maeno, Phys. Rev. Lett. 84 (2000) 2666.

[3] I.I. Mazin, D.J. Singh, Phys. Rev. Lett. 82 (1999) 4324.

[4] L. Bauernfeind, W. Widder, H. F. Braun, Physica C 254 (1995) 151.

[5] Y.S. Lee, J.S. Lee, K.W. Kim, T.W. Noh, Y. Jaejun, E.J. Choi, G. Cao, J.E. Crow, Europhys. Lett. 55 (2001) 280.

[6] P. Khalifah, K.D. Nelson, R. Jin, Z.Q. Mao, Y. Liu, Q. Huang, X.P.A. Gao, A.P. Ramirez, R.J. Cava, Nature 411 (2001) 669.

[7] R.S. Perry, L.M. Galvin, S.A. Grigera, L. Capogna, A.J. Schofield, A.P. Mackenzie, M. Chiao, S.R. Julian, S.I. Ikeda, S. Nakatsuji, Y. Maeno, C. Pfleiderer, Phys. Rev. Lett. 86 (2001) 2661.

[8] G.Cao, S. McCall, J.E. Crow, R.P. Guertin, Phys. Rev. Lett. 78 (1997) 1751.

[9] A. Callaghan, C.W. Moeller, R. Ward, Inorg. Chem. 5 (1966) 1572.

[10] P. Kostic, Y. Okada, N.C. Collins, Z. Schlesinger, J.W. Reiner, L. Klein, A. Kapitulnik, T.H. Geballe, M.R. Beasley, Phys. Rev. Lett. 81 (1998) 2498.

[11] J.M. Longo, P.M. Raccah, J.B. Goodenough, J. Appl. Phys. 39 (1968) 1327.

[12] J.L. Martinez, C. Prieto, J. Rodriguez-Carvajal, A. de Andres, M. Vallet-Regi, J.M. Gonzalez-Calbet, J. Magn. Magn. Mater. 140-144 (1995) 179.

[13] T. Kiyama, K. Yoshimura, K. Kosuge, H. Mitamura, T. Goto, J. Phys. Soc. Jpn. 68 (1999) 3372.

[14] K. Yoshimura, T. Imai, T. Kiyama, K.R. Thurber, A.W. Hunt, K. Kosuge, Phys. Rev. Lett. 83 (1999) 4397.

[15] T.C. Gibb, R. Greatrex, N.N. Greenwood, P. Kaspi, J. Chem. Soc. (Dalton Trans.) (1973) 1253.

[16] I.I. Mazin, D.J. Singh, Phys. Rev. B 56 (1997) 2556.

[17] I. Felner, I. Nowik, I. Bradaric, M. Gospodinov, Phys. Rev. B 62 (2000) 11332.

[18] G. Santi, T. Jarlborg, J. Phys: Condens. Matter 9 (1997) 9563.

[19] J. M. Wills, O. Eriksson, M. Alouani, D. L. Price in: H. Dreysse (Ed.) Electronic Structure and Physical Properties of Materials, Springer, Berlin, 2000, p.148

[20] J. P. Perdew, S. Burke, M. Ernzerhof, Phys. Rev. Lett. 77 (1996) 3865.
[21] H. Kobayashi, M. Nagata, R. Kanno, Y. Kawamoto, Mater. Res. Bull. 29 (1994) 1271.

[22] R. Vidya, P.Ravindran, A.Kjekshus, H.Fjellvåg, B.C.Hauback (unpublished).

[23] P. Ravindran, H. Fjellvåg, A. Kjekshus, A. Delin, O. Eriksson, Phys. Rev. B 65 (2002) 064445.

[24] G. Cao, S. McCall, M. Shepard, J.E. Crow, R.P. Buertin, Phys. Rev. B 56 (1997) 321.

[25] P. Ravindran,A. Kjekshus, H. Fjellvåg, P. James, L. Nordström, B. Johansson, O. Eriksson, Phys. Rev. B 63 (2001) 144409.

[26] J. B. Goodenough in:J. T. Lopuszanski J. T. Pekalski, J. Przystawa (Eds.) Magnetism in Metals and Metallic Compounds, Plenum, New York, 1974, p.35.

[27] H.Mukuda, K. Ishida, Y. Kitaoka, K. Asayama, R. Kanno, M. Takano, Phys. Rev. B 60 (1999) 12279.

[28] F. Fukunaga, N Tsuda, J. Phys. Soc. Jpn. 63 (1994) 3798.

[29] P. Ravindran, R. Asokamani, Bull. Mater. Sci. 20 (1997) 613.

[30] M. V. Rama Rao, V. G. Sathe, D. Sornadurai, B. Panigrahi, T. Shripathi, J. Phys. Chem. Solids 62 (2001) 797.

[31] J. S. Lee, Y. S. Lee, T. W. Noh, K. Char, J. Park, S.J. Oh, J.-H. Park, C. B. Eom, T. Takeda, R. Kanno, cond-mat/0107375 (2001).

[32] G. Cao, F. Freibert, J.E. Crow, J. Appl. Phys. 81 (1998) 3884 .

[33] J. B. Goodenough, Magnetism and the Chemical Bond, Wiley, New York, 1963.

[34] I. M. Bradaric, I. Felner, M. Gospodinov, condmat/0107170 (2001).

[35] R.K. Li, C. Greaves, Phys. Rev. B 62 (2000) 3811.

[36] M. Shepard, G. Cao, S. McCall, F. Freibert, J.E. Crow, J. Appl. Phys. 79 (1996) 4821.

[37] D. Chowdhury, Spin Glasses and Other Frustrated Systems, World Scientific, Singapore, 1986, p.7.

[38] K.H. Fischer, J.A. Hertz, Spin Glasses, Cambridge University Press, Cambridge, 1997, p.2

[39] M. Ito, Y. Yasui, M. Kanada, H. Harashna, S. Yoshii, K. Murata, M. Sato, H. Okumura K. Kakurai, J. Phys. and Chem. Solids 62 (2001) 337.

[40] G. Cao, S. McCall, J. Bolivar, M. Shepard, F. Freibert, P. Henning, J.E. Crow, T. Yuen, Phys. Rev. B 54 (1996) 15144 . 